sion in peripheral blood lymphocytes from patients with systemic lupus erythematosus. Autoimmunity. 29:111-120.

23. Kammer, G.M., Khan, I.U., and Malemud, C.J. 1994. Deficient type I protein kinase A isozyme activity in systemic lupus erythematosus $\mathrm{T}$ lymphocytes. J. Clin. Invest. 94:422-430.

24. Tada, Y., Nagasawa, K., Yamauchi, Y., Tsukamoto, H., and Niho, Y. 1991. A defect in the protein kinase $\mathrm{C}$ system in $\mathrm{T}$ cells from patients with systemic lupus erythematosus. Clin. Immunol. Immunopathol. 60:220-231.

25. Gergely, P., Jr., et al. 2002. Persistent mitochondrial hyperpolarization, increased reactive oxygen intermediate production, and cytoplasmic alkalinization characterize altered IL-10 signaling in patients with systemic lupus erythematosus. J. Immunol. 169:1092-1101.
26. Gergely, P., Jr., et al. 2002. Mitochondrial hyperpolarization and ATP depletion in patients with systemic lupus erythematosus. Arthritis Rheum. 46:175-190.

27. Yi, Y., McNerney, M., and Datta, S.K. 2000. Regulatory defects in $\mathrm{Cbl}$ and mitogen-activated protein kinase (extracellular signal-related kinase) pathways cause persistent hyperexpression of CD40 ligand in human lupus T cells. J. Immunol. 165:6627-6634.

28. Juang, Y.-T., Tenbrock, K., Nambiar, M.P., Gourley, M.F., and Tsokos, G.C. 2002. Defective production of functional $98-\mathrm{kDa}$ form of Elf-1 is responsible for the decreased expression of TCR z-chain in patients with systemic lupus erythematosus. J. Immunol. 169:6048-6055.

29. Kyttaris, V.C., Juang, Y.-T., Tenbrock, K., Weinstein, A., and Tsokos, G.C. 2004. Cyclic adenosine 5 '-monophosphate response element modula- tor is responsible for the decreased expression of c-fos and activator protein-1 binding in T cells from patients with systemic lupus erythematosus. J. Immunol. 173:3557-3563.

30. Wong, H.K., Kammer, G.M., Dennis, G., and Tsokos, G.C. 1999. Abnormal NF-kB activity in T lymphocytes from patients with systemic lupus erythematosus is associated with decreased p65-RelA protein expression. J. Immunol. 163:1682-1689.

31. Deng, C., et al. 2001. Decreased ras-mitogen-activated protein kinase signaling may cause DNA hypomethylation in T lymphocytes from lupus patients. Arthritis Rheum. 44:397-407.

32. Grolleau, A., Kaplan, M.J., Hanash, S.M., Beretta, L., and Richardson, B.C. 2000. Impaired translational response and increased protein kinase PKR expression in T cells from lupus patients. J. Clin. Invest. 106:1561-1568.

\title{
In hypertension, the kidney is not always the heart of the matter
}

\author{
Michael E. Mendelsohn
}

Molecular Cardiology Research Institute, Tufts-New England Medical Center, Boston, Massachusetts, USA.

\begin{abstract}
Blood pressure abnormalities are thought to originate from intrinsic changes in the kidney, a concept that has been largely unchallenged for more than 4 decades. However, recent molecular, cellular, and transgenic mouse studies support an alternative hypothesis: primary abnormalities in vascular cell function can also directly cause abnormalities of blood pressure. In this issue of the JCI, Crowley and coworkers describe the application of an elegant cross-renal transplant model to type $1 \mathrm{~A}$ angiotensin $\left(\mathrm{AT}_{1 \mathrm{~A}}\right)$ receptor-deficient mice and their wild-type littermates to explore the relative contributions of renal and extrarenal tissues to the low blood pressure seen in the $\mathrm{AT}_{1 \mathrm{~A}}$ receptor-deficient animals (see the related article beginning on page 1092). Their studies further support the emerging paradigm that primary abnormalities of the vasculature can make unique, nonredundant contributions to blood pressure regulation; the findings have potentially important implications for the ways we diagnose and treat blood pressure diseases in humans.
\end{abstract}

\section{Introduction}

Abnormalities of blood pressure, especially high blood pressure or hypertension, are widespread, cause extensive morbidity and mortality, and are insidious, because they rarely generate symptoms. But what causes hypertension? This simple question is remarkably difficult to answer, in large

Nonstandard abbreviations used: $\mathrm{AT}_{1 \mathrm{~A}}$, type $1 \mathrm{~A}$

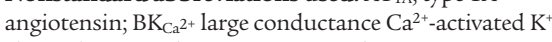
channel; $\mathrm{CO}$, cardiac output; $\mathrm{D}^{+} \mathrm{R}^{+}$, donor ${ }^{+}$recipient ${ }^{+}$; $\mathrm{IP}_{3}$, inositol triphosphate; MLC, myosin light chain; MLCK, MLC kinase; PKGI, cGMP-dependent protein kinase type I; PP1M, myosin phosphatase; RAS, reninangiotensin system; RGS2, regulator of G protein signaling 2; SVR, systemic vascular resistance.

Conflict of interest: The author has declared that no conflict of interest exists.

Citation for this article: J. Clin. Invest. 115:840-844 (2005). doi:10.1172/JCI200524806. part because blood pressure, which is measurable only in intact animals, is an integrated value determined by contributions from a complex mix of rheologic, renal, neural, vascular, and hormonal variables. It is therefore difficult to distinguish primary or causal factors for abnormal blood pressure from those responses that are secondary to the changes in pressure.

Blood pressure is proportional to 2 fundamental hemodynamic factors: cardiac output $(\mathrm{CO})$ and systemic vascular resistance $(\mathrm{SVR})$, and the equation $\mathrm{BP}=\mathrm{CO} \times \mathrm{SVR}$ is taught to every first-year physiology student. The pioneering studies of Guyton and colleagues provide abundant support for the central, causal role of abnormal renal sodium handling in the etiology of hypertension $(1,2)$. This work emphasiz- es the primacy of the kidney's "pressure control system," which is recruited when systemic pressure is altered and causes a renally mediated increase or decrease in blood volume to establish long-term blood pressure control. Elegant genetic studies published over the past decade have provided further strong support for the hypothesis that alterations of net salt balance underlie abnormalities of blood pressure in humans (3). But are the alterations in renal salt balance that cause abnormal blood pressure always direct, as has been argued for many years, or could renal alterations in some cases be indirect or secondary to primary abnormalities of nonrenal tissues? Does the kidney always cause abnormal blood pressure by altering blood volume, which in turn alters $\mathrm{CO}$ and SVR? Or do intrinsic abnormalities of the vasculature in some cases give rise to a primary abnormality of SVR, causing hypertension? Surprisingly, we do not yet know the answer to this simple dilemma.

In this issue of the JCI, Crowley and colleagues (4) describe cross-renal transplantation studies with type $1 \mathrm{~A}$ angiotensin $\left(\mathrm{AT}_{1 \mathrm{~A}}\right)$ receptor-deficient $\left(\right.$ Agtrla $\left.\mathrm{a}^{-/-}\right)$mice and their wild-type littermates. Using stateof-the-art approaches to examine mouse physiology and sophisticated, technically demanding microsurgical techniques, their study provides new and important insights into the relative importance of renal and nonrenal $\mathrm{AT}_{1}$ receptors in deter- 
Primary abnormality of VSMC function

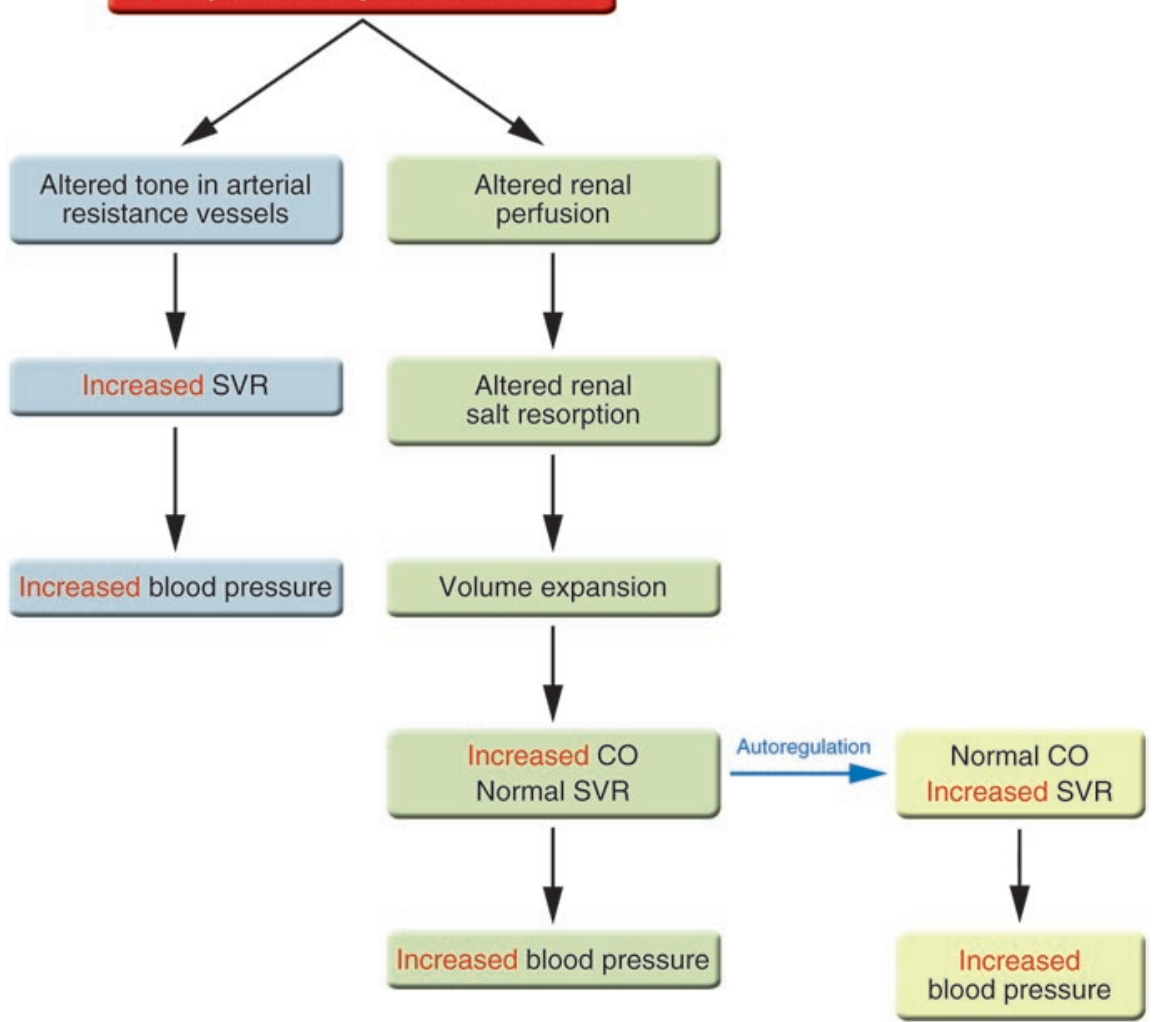

Figure 1

An alternative model for the pathogenesis of hypertension. According to the prevailing model for hypertension, a renal abnormality in salt handling is the initiating factor. In the alternative model discussed here, hypertension arises de novo from primary, intrinsic abnormalities of the VSMC proteins that regulate contractile state. At least 2 mechanisms can be envisioned. Alterations in the set point of resting vascular tone of the resistance arteries could modify blood pressure by directly increasing SVR. Alternatively, intrinsic abnormalities in the VSMCs of renal vessels could alter renal vascular tone and perfusion, secondarily creating the recognized effects on renal function that promote and/or sustain blood pressure elevations. In either case, a focus on those regulatory proteins in the final common pathway regulating VSMC tone suggests new diagnostic and therapeutic approaches to abnormalities of blood pressure. Figure modified with permission from Cell (3).

mining blood pressure. $\mathrm{AT}_{1 \mathrm{~A}}$ receptor-deficient mice are hypotensive, and their lower blood pressures have been attributed to renal changes in the function of the reninangiotensin system (RAS). As the authors explain, their experiments were designed to explore the relative contributions of individual tissue compartments to the low blood pressure seen in $\mathrm{AT}_{1 \mathrm{~A}}$-deficient mice, since there is at present a surprising lack of direct proof of the primacy of the kidney in the regulation of blood pressure by RAS.

In the current study, 4 experimental groups of mice were created, and mice in all 4 groups received a new kidney by transplantation (4). The 4 groups studied included wild-type animals that received a wild-type kidney (group I or donor ${ }^{+}$ recipient $\left.t^{+}\left[\mathrm{D}^{+} \mathrm{R}^{+}\right]\right)$; wild-type animals that received an $\mathrm{AT}_{1 \mathrm{~A}}$ receptor-knockout mouse kidney (group II or $\mathrm{D}^{-} \mathrm{R}^{+}$); $\mathrm{AT}_{1 \mathrm{~A}}$ receptor-knockout mice that received a wild-type kidney (group III or $\mathrm{D}^{+} \mathrm{R}^{-}$); and $\mathrm{AT}_{1 \mathrm{~A}}$ receptor-knockout mice that received an $\mathrm{AT}_{1 \mathrm{~A}}$ receptor-knockout mouse kidney (group IV or $\mathrm{D}^{-} \mathrm{R}^{-}$). Mice in all 4 groups also underwent implantation of radiotelemetry transmitters 6-8 days after kidney transplant procedure to monitor blood pressure. Untransplanted wild-type and $\mathrm{AT}_{1 \mathrm{~A}}$ receptor-knockout mice also received blood pressure transmitters and served as additional controls.

The results of these experiments are both highly informative and provocative. Blood pressures in $\mathrm{D}^{+} \mathrm{R}^{+}$mice were no different from those in nontransplanted, wild-type mice, which confirmed that the transplant procedure itself does not significantly alter systemic pressure (4). $\mathrm{D}^{-} \mathrm{R}^{+}$mice had significant reductions in blood pressure, as expected, which supports the importance of the kidney in determining systemic pressure. However, the $\mathrm{D}^{+} \mathrm{R}^{-}$mice had similar, significant reductions in blood pressure. This is the "punch line" of the article: nonrenal tissues have an equal and independent role in determining blood pressure in these mice. Furthermore, the presence of intact $\mathrm{AT}_{1 \mathrm{~A}}$ receptor signaling in the kidneys of $\mathrm{D}^{+} \mathrm{R}^{-}$mice was insufficient to compensate for the absence of $\mathrm{AT}_{1 \mathrm{~A}}$ receptors in their nonrenal tissues. The authors performed additional studies to determine whether lower aldosterone production in the $\mathrm{D}^{+} \mathrm{R}^{-}$mice might account for their lower blood pressures, using an aldosterone clamping approach in which mice received steady infusions of this hormone. However, despite supraphysiological and equivalent aldosterone levels, blood pressures remained significantly lower in the $\mathrm{D}^{+} \mathrm{R}^{-}$mice compared with $\mathrm{D}^{+} \mathrm{R}^{+}$mice, which demonstrates the critical importance of nonrenal tissues in the determination of blood pressure.

These cross-transplantation studies in $\mathrm{AT}_{1 \mathrm{~A}}$ receptor-deficient mice (4) do not precisely localize the nonrenal tissues responsible for determining blood pressure, but they provide strong hints that one such tissue is the vasculature. Sympathetic nervous system activation is thought to be important to the initiation and maintenance of high blood pressure and to contribute to hypertension (reviewed in refs. $5,6)$. However, renal norepinephrine levels (an accepted measure of renal sympathetic nerve activity) were reduced equally in the $\mathrm{D}^{+} \mathrm{R}^{+}$and $\mathrm{D}^{+} \mathrm{R}^{-}$mice, which indicates that altered sympathetic activity is an unlikely explanation for the reduced blood pressures observed in $\mathrm{D}^{+} \mathrm{R}^{-}$mice. The chronic reduction in renal norepinephrine levels confirms the persistent absence of sympathetic innervation in the transplanted kidneys and supports the notion that the reduced blood pressure observed in the $\mathrm{D}^{+} \mathrm{R}^{-}$mice results from direct vascular effects of $\mathrm{AT}_{1 \mathrm{~A}}$ receptor deficiency.

\section{Primary vascular abnormalities as a cause of abnormal blood pressure}

Though blood vessel tone is a major determinant of blood pressure, and abnormalities in vascular tone are common in hypertensive and ischemic disorders, the notion that abnormalities of vascular tone may in 


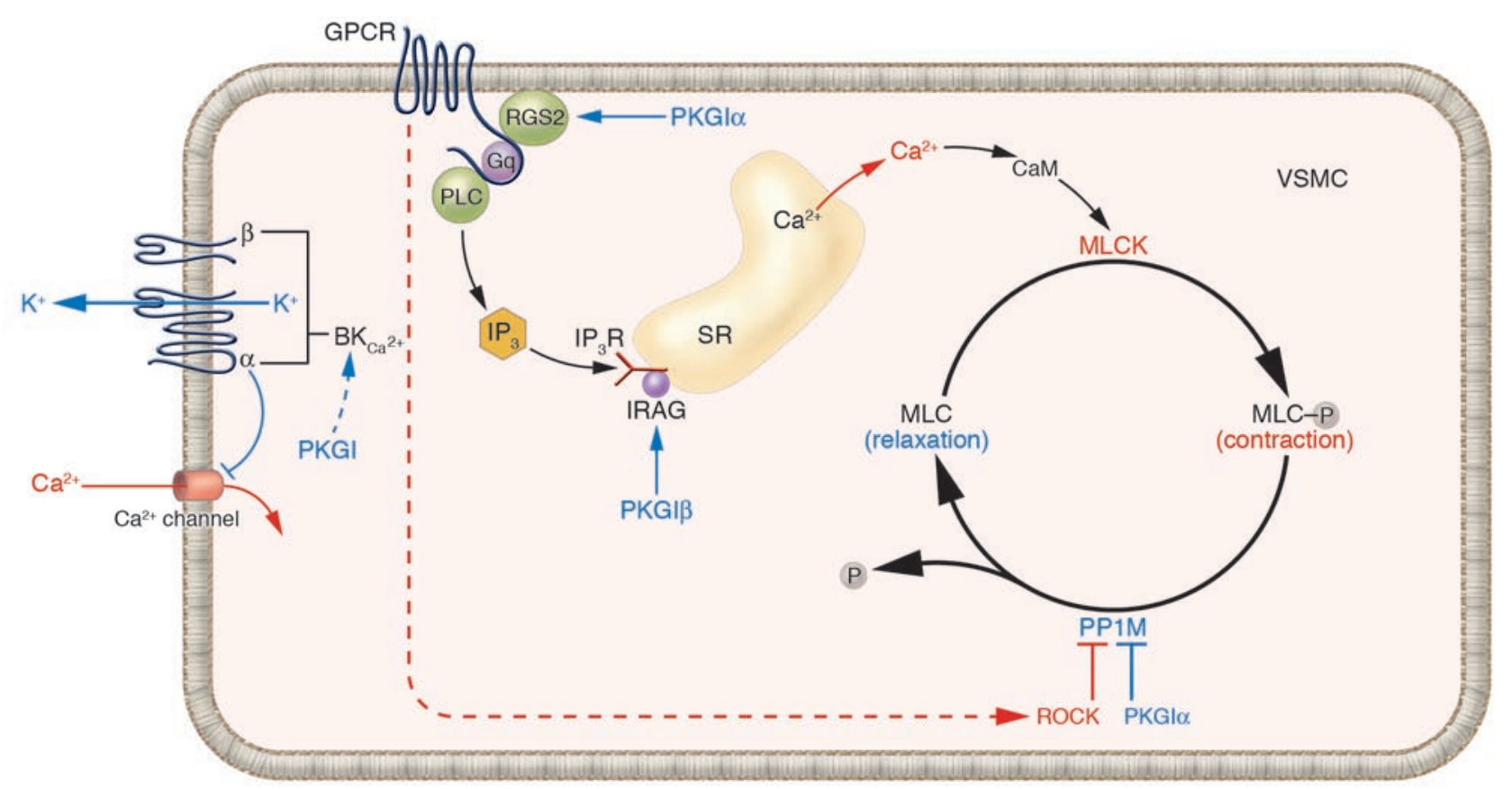

\section{Figure 2}

Vascular tone is dynamically regulated by MLC phosphorylation and dephosphorylation in VSMCs. Increases in VSMC intracellular calcium level via receptor-activated (pharmacomechanical) or ion channel-activated (electromechanical) pathways lead to MLCK activation. MLCK phosphorylates MLCs (MLC-P), activating the myosin ATPase, actinomyosin cross-bridging, and an increase in tension. PP1M dephosphorylates MLC-P, decreasing cell tension. PP1M is activated by the NO and nitrovasodilator effector PKGI, which has 2 isoforms (PKGl $\alpha$ and PKGI $\beta$ ). RGS2, which is essential for normal blood pressure, causes VSMC relaxation by attenuating Gq protein-coupled receptor activation and associated rises in intracellular calcium concentration; it too is activated by PKGl $\alpha$. PP1M is inhibited by Rho/Rho kinase (ROCK). The calcium-activated potassium channel, $\mathrm{BK}_{\mathrm{Ca}^{2+}}$, is activated by $\mathrm{PKG}$ and by local calcium sparks, hyperpolarizing the cell, decreasing calcium entry, and decreasing MLCK activity. This shifts the equilibrium between MLC and MLC-P, causing relaxation. Blue, relaxant pathway; red, contractile pathway. GPCR, G protein-coupled receptor; $\mathrm{IP}_{3} \mathrm{R}, \mathrm{IP}_{3}$ receptor; SR, sarcoplasmic reticulum; PLC, phospholipase C; CaM, calmodulin; IRAG, IP $\mathrm{I}_{3}$-associated cGMP kinase substrate.

some cases actually cause high or low blood pressure remains unproven. Work over the past 5 years, including that of Crowley et al. discussed here (4), supports the hypothesis that VSMC abnormalities that alter the intrinsic contractile state of the cell can directly cause abnormal vascular tone and disorders of blood pressure regulation, including hypertension. Put differently, according to this hypothesis, primary vasodilatation or vasoconstriction is alone sufficient to cause hypotension or hypertension, respectively. Vasoconstriction could produce hypertension in at least 2 different ways (Figure 1). Hypertension could arise de novo from alterations in the set point of resting vascular tone in the resistance vessels that regulate blood pressure. Alternatively, intrinsic abnormalities in the VSMCs of renal vessels could alter renal vacular tone and perfusion, thus secondarily creating the recognized changes in salt handling by the kidney that promote and/or sustain blood pressure elevations. In either case, however, the fundamental abnormality resides in the

critical VSMC proteins that regulate the contractile state of the cell, which focuses our attention on different diagnostic and therapeutic targets.

\section{Regulation of smooth muscle cell tone}

VSMCs are the principal cell of the blood vessel wall, and VSMC contractile state is dynamically and rapidly regulated by hor-

\section{Table 1}

Mouse models of vascular contractile dysfunction and hypertension

\section{Mouse model}

$\mathrm{BK}_{\mathrm{Ca}}{ }^{2+}$ channel $\beta 1$ subunit KO

Estrogen receptor $\beta$ KO

VSMC Sur2 K(ATP) channel KO

eNOS KO

RGS2 KO

PKGI KO

PKGl $\alpha$ leucine zipper mutant knockin monal and neural inputs. However, the basal state of VSMC tone is determined by a small number of proteins that are part of a final common pathway regulating force generation by the cell's actinomyosinbased contractile apparatus. VSMC contraction is initiated by a rise in intracellular calcium, which can occur as a result of either: entry of extracellular calcium into the cell via calcium channels following

HTN, hypertension; Sur2, sulfonylurea receptor subunit 2. 
membrane depolarization; or mobilization of intracellular calcium stores following activation of membrane receptors by contractile agonists (Figure 2) (reviewed in ref. $7)$. When intracellular calcium rises, myosin light chain kinase (MLCK) is activated (Figure 2) and phosphorylates the regulatory myosin light chains (MLCs), which causes contraction (7). Conversely, when MLCs are dephosphorylated by the critical regulatory protein myosin phosphatase (PP1M), relaxation occurs (reviewed in refs. 7,8$)$. Thus, smooth muscle contractile state is determined by the state of MLC phosphorylation, which is directly determined by the balance of MLCK and PP1M activities. It is not surprising that MLCK and PP1M are themselves highly regulated and that alterations in their activities can shift resting VSMC tone. Proteins regulating VSMC intracellular calcium levels and MLC phosphorylation state are therefore good candidates for primary regulators of blood pressure.

Abnormalities in contractile pathway proteins can cause primary changes in VSMC tone and hypertension. Voltage-gated calcium channels, which allow calcium ions to enter VSMCs, are sensitive to the resting potential of the cell and therefore regulated by potassium channels, which make major contributions to determining cellular potential. In VSMCs, the large conductance $\mathrm{Ca}^{2+}$-activated $\mathrm{K}^{+}$channel $\left(\mathrm{BK}_{\mathrm{Ca}^{2+}}\right)$ is an important primary regulator of vascular relaxation (9). $\mathrm{BK}_{\mathrm{Ca} 2+}$ is activated by local calcium sparks (reviewed in ref. 10) and by cGMP-dependent protein kinase (PKG) (11); this results in hyperpolarization of the cell, which decreases calcium entry and causes relaxation. VSMCs from mice with several potassium channel abnormalities display abnormal vascular contraction and hypertension (12-14) (Table 1). Many contractile agonists work by activating $\mathrm{G}$ protein-coupled receptors, which trigger mobilization of intracellular calcium and activation of MLCK (15). These receptors also activate Rho/Rho kinase, which directly inhibits PP1M, augmenting contraction (8) (Figure 2). A recently recognized class of Rho kinase inhibitors lowers blood pressure (16), and one such compound, fasudil, shows promise in clinical trials as a new antihypertensive and vasospastic therapy $(17,18)$. It is also worth reconsidering the mechanisms of action of molecules we have assumed work solely through renal mechanisms to cause hypertension, such as the mineralocorticoid receptor $(19,20)$.
VSMC relaxation is also tightly regulated and the NO-cGMP-cGMP-dependent protein kinase type I (NO-cGMP-PKGI-dependent) pathway contains molecules that also are good candidates for primary vascular regulators of blood pressure. Mice lacking eNOS have hypertension (21), and though the mechanism of abnormal blood pressure regulation in these animals is not yet understood, it likely involves PKGI (Figure 2 ). The endogenous vasodilator $\mathrm{NO}$ and related compounds cause vascular relaxation by activating PKGI $\alpha$, which binds directly to and activates PP1M, reducing MLC phosphorylation and causing VSMC relaxation (22) (Figure 2). PKGI $\alpha$ also can relax smooth muscle cells by direct interaction with the regulator of $G$ protein signaling 2 (RGS2), a second PKGI substrate. RGS2 terminates signaling by Gq-coupled contractile agonist receptors, which mediate the actions of many physiologically important vascular contractile agonists (23). RGS2-deficient mice have abnormal vascular contraction and relaxation and are hypertensive $(23,24)$. The PKGI $\beta$ isoform regulates release of calcium from intracellular stores, and mice lacking the PKGI $\beta$ target protein inositol triphosphate receptor-associated cGMP kinase substrate (IRAG) have abnormal vascular relaxation $(25,26)$. Mice fully lacking PKGI (27) or with PKGI mutations that prevent the PKGI-PP1M interaction (28) also are hypertensive (Table 1).

The hypotensive $\mathrm{AT}_{1 \mathrm{~A}}$ receptor-deficient mice studied by Crowley et al. (4) represent a loss-of-function model. It will also be important to apply the cross-renal transplantation approach to mice with single gene abnormalities that cause hypertension (Table 1). More studies of mice harboring VSMC-specific changes in candidate blood pressure-determining genes are also needed. Ultimately, however, nonrenal candidate genes for blood pressure determination will need to be explored in humans. Careful scrutiny of genomic associations and polymorphisms in candidate genes will be required. Several hypertensionassociated loci that have been identified in genome-wide linkage studies contain candidate genes that we and others are currently exploring, including the Rho kinases (ROCK1, located at chromosome18q11.2; and ROCK2, at 2p24) $(29,30)$ and the BK channel $\beta$ subunit (on 5q34) $(31,32)$. Identification of new, nonrenal genes capable of determining blood pressure in humans would have important implications for diagnosis and therapy of a wide spectrum of cardiovascular disorders.

Address correspondence to: Michael E. Mendelsohn, Molecular Cardiology Research Institute, Tufts-New England Medical Center, 750 Washington St., Box 080, Boston, Massachusetts 02111, USA. Phone: (617) 636-9370; Fax: (617) 636-1444; E-mail: MMendelsohn@tufts-nemc.org.

1. Guyton, A.C., et al. 1972. Arterial pressure regulation. Overriding dominance of the kidneys in longterm regulation and in hypertension. Am. J. Med. 52:584-594.

2. Guyton, A.C. 1991. Blood pressure control - special role of the kidneys and body fluids. Science. 252:1813-1816.

3. Lifton, R.P., Gharavi, A.G., and Geller, D.S. 2001. Molecular mechanisms of human hypertension [review]. Cell. 104:545-556.

4. Crowley, S.D., et al. 2005. Distinct roles for the kidney and systemic tissues in blood pressure regulation by the renin-angiotensin system. J. Clin. Invest. 115:1092-1099. doi:10.1172/JCI200523378.

5. Ferrario, C.M., and Averill, D.B. 1991. Do primary dysfunctions in neural control of arterial pressure contribute to hypertension? Hypertension. 18(Suppl. 3):I38-I51.

6. Sved, A.F., Ito, S., and Sved, J.C. 2003. Brainstem mechanisms of hypertension: role of the rostral ventrolateral medulla. Curr. Hypertens. Rep. 5:262-268.

7. Somlyo, A.P., and Somlyo, A.V. 1994. Signal transduction and regulation in smooth muscle. Nature. 372:231-236.

8. Hartshorne, D.J., Ito, M., and Erdodi, F. 2004. Role of protein phosphatase type 1 in contractile functions: myosin phosphatase. J. Biol. Chem. 279:37211-37214.

9. Brayden, J.E., and Nelson, M.T. 1992. Regulation of arterial tone by activation of calcium-dependent potassium channels. Science. 256:532-535.

10. Jaggar, J.H., and Nelson, M.T. 2000. Calcium sparks in smooth muscle. Am.J. Physiol., Cell Physiol. 278:C235-C256.

11. Robertson, B.E., Schubert, R., Hescheler, J., and Nelson, M.T. 1993. cGMP-dependent protein kinase activates $\mathrm{Ca}$-activated $\mathrm{K}$ channels in cerebral artery smooth muscle cells. Am. J. Physiol., Cell Physiol. 265:C299-C303.

12. Brenner, R., et al. 2000. Vasoregulation by the B1 subunit of the calcium-activated potassium channel. Nature. 407:870-876.

13. Zhu, Y., et al. 2002. Abnormal vascular function and hypertension in mice deficient in estrogen receptor $\beta$. Science. 295:505-508.

14. Chutkow, W.A., et al. 2002. Episodic coronary artery vasospasm and hypertension develop in the absence of Sur2 K(ATP) channels. J. Clin. Invest. 110:203-208. doi:10.1172/JCI200215672.

15. Demoliou-Mason, C.D. 1998. G-protein-coupled receptors in vascular smooth muscle cells. Biol. Signals. 7:90-97.

16. Uehata, M., et al. 1997. Calcium sensitization of smooth muscle mediated by a Rho-associated protein kinase in hypertension. Nature. 389:990-994.

17. Masumoto, A., et al. 2001. Possible involvement of rho-kinase in the pathogenesis of hypertension in humans. Hypertension. 38:1307-1310.

18. Hirooka, Y., Shimokawa, H., and Takeshita, A. 2004. Rho-kinase, a potential therapeutic target for the treatment of hypertension. Drug News Perspect. 17:523-527.

19. Geller, D.S., et al. 2000. Activating mineralocorticoid receptor mutation in hypertension exacerbated by pregnancy. Science. 289:119-123. 
20. Jaffe, I.Z., and Mendelsohn, M.E. 2005. Angiotensin II and aldosterone regulate gene transcription via functional mineralocortocoid receptors in human coronary artery smooth muscle cells. Circ. Res. doi:10.1161/01.RES.0000159937.05502.d1.

21. Huang, P.L., et al. 1995. Hypertension in mice lacking the gene for endothelial nitric oxide synthase. Nature. 377:239-242.

22. Surks, H.K., et al. 1999. Regulation of myosin phosphatase by a specific interaction with cGMPdependent protein kinase I $\alpha$. Science. 286:1583-1587.

23. Tang, M., et al. 2003. Regulator of G-protein signaling 2 mediates vascular smooth muscle relaxation and blood pressure. Nat. Med. 9:1506-1512.

24. Le, T.H., and Coffman, T.M. 2003. RGS2: a "turnoff” in hypertension. J. Clin. Invest. 111:441-443.
doi:10.1172/JCI200317836.

25. Schlossmann, J., et al. 2000. Regulation of intracellular calcium by a signalling complex of IRAG, $\mathrm{IP}_{3}$ receptor and cGMP kinase I $\beta$. Nature. 404:197-201

26. Geiselhoringer, A., et al. 2004. IRAG is essential for relaxation of receptor-triggered smooth muscle contraction by cGMP kinase. EMBO J. 23:4222-4231.

27. Pfeifer, A., et al. 1998. Defective smooth muscle regulation in cGMP kinase I-deficient mice. $E M B O J$. 17:3045-3051

28. Mendelsohn, M.E. 2005. Molecular mechanisms regulating vascular smooth muscle cell tone and blood pressure [abstract]. Keystone Symposia: The Cellular Biol ogy of Atherosclerosis. Keystone, Colorado, USA.

29. Levy, D., et al. 2000. Evidence for a gene influenc- ing blood pressure on chromosome 17: genome scan linkage results for longitudinal blood pressure phenotypes in subjects from the Framingham Heart Study. Hypertension. 36:477-483.

30. Angius, A., et al. 2002. A new essential hypertension susceptibility locus on chromosome 2p24-p25, detected by genomewide search. Am.J. Hum. Genet. 71:893-905.

31. Krushkal, J., et al. 1999. Genome-wide linkage analyses of systolic blood pressure using highly discordant siblings. Circulation. 99:1407-1410.

32. Fernandez-Fernandez, J.M., et al. 2004. Gain-offunction mutation in the KCNMB1 potassium channel subunit is associated with low prevalence of diastolic hypertension. J. Clin. Invest. 113:1032-1039. doi:10.1172/JCI200420347.

\title{
Ikaros transcription factors: flying between stress and inflammation
}

\author{
George P. Chrousos ${ }^{1,2}$ and Tomoshige Kino²
}

${ }^{1}$ First Department of Pediatrics, University of Athens, Athens, Greece. 2Reproductive Biology and Medicine Branch, National Institute of Child Health and Human Development, NIH, Bethesda, Maryland, USA.

\begin{abstract}
The hypothalamic-pituitary-adrenal axis (a major component of the stress system) and the immune system contribute to the maintenance of homeostasis at rest and during stress. Because of their essential roles for the survival of self and species, the activities of these systems have evolutionarily developed in parallel and are intertwined at many levels. In this issue of the JCI, Ezzat et al. demonstrate that Ikaros, a differentiation factor of leukocyte lineage, also influences the maturation of the fetal pituitary corticotroph and, hence, the secretion of adrenocorticotropic hormone before and after birth (see the related article beginning on page 1021). These results indicate that Ikaros is an ontogenetic and phylogenetic integrator of the stress and immune systems and that abnormalities in its function may produce endocrine and/or immune pathologies.
\end{abstract}

\section{The stress and immune systems influence each other's activity}

The stress and immune systems play essential roles in the maintenance of resting and stress-related homeostasis (1-3). The former regulates behavioral, cardiovascular, metabolic, and immune homeostasis, so that individuals can survive under various stressful conditions. The latter distinguishes between self and non-self and between injurious and noninjurious agents and protects the individual from external and

Nonstandard abbreviations used: ACTH, adrenocorticotrophic hormone; AVP, arginine vasopressin; $\mathrm{CRH}$, corticotropin-releasing hormone; GR, glucocor ticoid receptor; HPA, hypothalamic-pituitary-adrenal; $\alpha$-MSH, $\alpha$-melanocyte-stimulating hormone; POMC, proopiomelanocortin.

Conflict of interest: The authors have declared that no conflict of interest exists.

Citation for this article: J. Clin. Invest. 115:844-848 (2005). doi:10.1172/JCI200524886 internal pathogens by eliminating them via several discrete mechanisms. As these 2 systems have been very important for survival of the self and species, they have evolved in parallel, sharing regulatory and mediatory molecules and mutually influencing each other's activities in both a cell- and a tissueand time-specific manner.

When the stress and immune systems are activated during inflammation, their interaction is particularly evident in the relationship between the HPA axis and the inflammatory/immune reaction (Figure 1 and Table 1). The HPA axis, together with the arousal and autonomic systems, form the stress system. The central components of this system are located in the hypothalamus and brain stem. These centers, the paraventricular nucleus (PVN) and the locus caeruleus/norepinephrine system, receive signals of internal and external changes, respectively, from both the central nervous system and distant organs. The PVN transduces these signals to the pituitary gland by secreting corticotropin-releasing hormone (CRH) and arginine vasopressin (AVP) into the hypophyseal portal system. The pituitary gland, in particular its component corticotroph cells, is synergistically activated by these neuropeptides and secretes adrenocorticotropic hormone (ACTH) into the systemic circulation, stimulating the cortices of the adrenal glands to produce glucocorticoids. These systemic end-effector hormones interact with the ubiquitous glucocorticoid receptors (GRs) in target tissues and alter the biologic activities of the brain and most organs and tissues, including the immune system.

In general, activation of the HPA axis alters the activity of certain important facets of the immune system, mainly through the activity of glucocorticoids and their intracellular signaling system $(2,3)$. These hormones, in fact, exert quite diverse actions, both inhibitory and stimulatory, on nearly all aspects of the immune reaction, including the innate, cellular, and humoral immune response (4). Indeed, glucocorticoids inhibit the production of proinflammatory cytokines as well as other mediators of inflammation, such as histamine, bradykinin, serotonin, and many prostanoids, during both the innate and adaptive immune responses. The same hormones, however, stimulate the production of several antiinflammatory 